\title{
Addressing the Issue of Systematic Errors in a Regional Climate Model
}

\author{
VASUBANDHU MisRa \\ Center for Ocean-Land-Atmosphere Studies, Institute of Global Environment and Society, Inc., Calverton, Maryland
}

(Manuscript received 6 October 2005, in final form 30 June 2006)

\begin{abstract}
A methodology is proposed in which a few prognostic variables of a regional climate model (RCM) are strongly constrained at certain wavelengths to what is prescribed from the bias-corrected atmospheric general circulation model (AGCM; driver model) integrations. The goal of this strategy is to reduce the systematic errors in a RCM that mainly arise from two sources: the lateral boundary conditions and the $\mathrm{RCM}$ errors. Bias correction (which essentially corrects the climatology) of the forcing from the driving model addresses the former source while constraining the solution of the RCM beyond certain relatively large wavelengths in the regional domain [also termed as scale-selective bias correction (SSBC)] addresses the latter source of systematic errors in RCM. This methodology is applied to experiments over the South American monsoon region. It is found that the combination of bias correction and SSBC on the nested variables of divergence, vorticity, and the log of surface pressure of an RCM yields a major improvement in the simulation of the regional climate variability over South America from interannual to intraseasonal time scales. The basis for such a strategy is derived from a systematic empirical approach that involved over 100 regional seasonal climate integrations.
\end{abstract}

\section{Introduction}

The utility and use of regional climate models (RCMs) has grown immensely over the years (Leung et al. 2003), which has made them indispensable tools for climate studies. The seminal work of Denis et al. (2002) clearly establishes the benefit of dynamic downscaling from coarse-resolution analysis. They showed that dynamic downscaling has the ability to reproduce realistic small-scale features in a "perfect prognosis" approach. In a related study, Antic et al. (2004) showed that complex topography and coastline features have a strong impact on the reproducibility of small-scale climate features that a RCM can resolve. But, with the growing use of RCMs it is also becoming apparent that the RCM integrations are limited by the errors in forcing from the lateral boundary conditions (LBCs; Risbey and Stone 1996; Noguer et al. 1998; Christensen et al. 1998; Menendez et al. 2001; Misra et al. 2003). Furthermore, Christensen et al. (1998) indicate that the influence of

Corresponding author address: Vasubandhu Misra, Center for Ocean-Land-Atmosphere Studies, Institute of Global Environment and Society, Inc., 4041 Powder Mill Road, Suite 302, Calverton, MD 20705.

E-mail: misra@cola.iges.org

DOI: $10.1175 / J C L I 4037.1$

(C) 2007 American Meteorological Society the systematic errors in the LBC on the evolution of the regional climate in a RCM amplify as the resolution of the RCM is increased. Therefore, it is appropriate to address the issue of the systematic errors in RCMs.

Misra and Kanamitsu (2004, hereafter MK04) proposed a novel approach of anomaly nesting (AN) to avoid the influence of systematic errors from the AGCM on the RCM integration. In that study, the climatology of the driving AGCM was replaced with the corresponding climatology of the National Centers for Environmental Prediction (NCEP) reanalysis, while the anomalies of the atmospheric GCM (AGCM) were retained. This led to a significant improvement in the seasonal simulation and intraseasonal variability in the RCM integrations. In another related study, Kanamaru and Kanamitsu (2007, hereafter KK07) used the approach of scale-selective bias correction (SSBC) to show that the RCM simulations forced with NCEP reanalysis can be further improved. This scheme introduced three steps to maintain the large scale prescribed from the driver model to an RCM. They are

- damping the spectral time tendencies of the wind components in the RCM strongly toward the base field tendency (supplied from the driving reanalysis) at the longest waves in the regional domain,

- setting the area-averaged deviations of the RCM- 
predicted values of $T$ and $q$ from the corresponding base field values to zero, and

- setting the perturbation of the natural log of surface pressure $\left(\ln p_{s}\right)$ to the natural $\log$ of the mean difference of the surface pressure elevation between the $\mathrm{RCM}$ and the base field (obtained from the coarse reanalysis). This is to correct the anomalous pressure gradients that get introduced due to the use of separate orography in the coarse-resolution driver model and the finer resolution in the RCM.

Using this methodology of SSBC on the regional spectral model (RSM; Juang and Kanamitsu 1994; Juang et al. 1997), forced with NCEP reanalysis, showed significant improvements in their regional climate simulation over the continental United States. They argued that the SSBC scheme improves even the small-scale processes since the small-scale dynamics in the regional model are not influenced by the large-scale errors. As an example they demonstrate precipitation physics is improved through SSBC. They illustrate that the wet (dry) bias in the winter (summer) season of their control model run is ameliorated by SSBC. A similar method of forcing the large-scale flow in the interior domain of an RCM involves spectral nudging, as shown by von Storch et al. (2000) and Miguez-Macho et al. (2004).

In this paper we introduce an approach that combines the methodologies of AN proposed in MK04 and the SSBC that follows from KK07 to further improve upon seasonal and intraseasonal simulations from the $\mathrm{RCM}$. However, it is important to state that there are deviations from SSBC originally proposed in KK07 to accommodate the nature of the large-scale forcing to the regional model in this study. This will become more apparent when the methodology and the results are discussed.

We recognize that there are essentially two sources of systematic errors in a RCM integration, namely, that emanating from the lateral boundary forcing and that from the RCM itself. The former source includes errors that arise from the inadequacies of the coarseresolution driver model. The RCM errors include those that arise from the interpolation in space and time of the lateral boundary forcing, their empirical handling in the "sponge zone," the approximations and empiricism involved in the parameterization schemes, and the discretized advection and time integration methodologies. The errors in the lateral boundary forcing are partially addressed by AN, while SSBC addresses the errors arising from the drift in the RCM. This proposed methodology is tested here with the RSM that follows from Juang et al. (1997) and Misra et al. (2002). However, this methodology can easily be adapted to any other RCM. Furthermore, to draw useful inferences from this study we test this proposed methodology over the South American summer monsoon (SASM) domain so that we can compare with some of our past work (MK04; Misra 2004, 2005).

The mathematical problem of the LBCs in a RCM is ill posed, and trouble is therefore inevitable (Warner et al. 1997; von Storch et al. 2000; Staniforth 1997). Most methods to ameliorate the influence of the LBC on the integration of the RCM are empirical in nature (Marbaix et al. 2003). In this study we adopt a systematic empirical approach where we analyze the model results with many different combinations of the LBC forcing to arrive at a configuration that yields the best seasonal and intraseasonal simulations of the SASM. Although the approach is empirical, the final solution arrived at in this study is consistent with the approach adopted in data assimilation schemes. In data assimilation schemes, the weights given to observations and model first -guess field vary with the quality, density, and type of observations. Similarly, here we refine AN and SSBC in such a way that some variables are corrected for their bias, while others are allowed to evolve under the influence of these corrections. This refinement is arrived empirically from conducting over 100 experiments with the RCM.

In the following section we briefly describe the models used in this study followed by a discussion of the methodology. In section 4 we outline the experiments followed by discussion of results in section 5, with concluding remarks and a summary in section 6 .

\section{Model description}

For this study we have adopted the regional spectral model following Juang and Kanamitsu (1994), Juang et al. (1997), and Misra et al. (2002). The RSM predicts the total field of zonal wind $(u)$, meridional wind $(v)$, temperature $(T)$, natural $\log$ of surface pressure $\left(\ln p_{s}\right)$, and specific humidity $(q)$. A brief outline of the physics of the RSM is provided in Table 1. In appendix A we provide a mathematical description of the perturbation filtering that is unique to RSM.

The RSM is nested into the Center for Ocean-LandAtmosphere Studies (COLA) AGCM at T42 spectral truncation. The COLA AGCM has been extensively used for seasonal climate integrations over the region (Misra et al. 2003; Misra 2005). The outline of the physics of the COLA AGCM is provided in Table 1. The vertical resolution of the AGCM and the RSM are identical. Both the RSM and the COLA AGCM have a terrain-following $(\sigma)$ vertical coordinate system. 
TABLE 1. Outline of the NCEP reanalysis and the COLA AGCM models.

\begin{tabular}{|c|c|c|}
\hline Feature & RSM & COLA AGCM \\
\hline Deep convection & $\begin{array}{l}\text { Relaxed Arakawa-Schubert scheme } \\
\text { (Moorthi and Suarez 1992) }\end{array}$ & $\begin{array}{l}\text { Relaxed Arakawa-Schubert scheme } \\
\quad \text { (Moorthi and Suarez 1992) }\end{array}$ \\
\hline Planetary boundary layer & Hong and Pan (1996) & Mellor and Yamada (1982) \\
\hline Longwave radiation & Fels and Schwarzkopf (1975) & Harshvardhan et al. (1987) \\
\hline Shortwave radiation & Chou (1992) & Davies (1982) \\
\hline Shallow convection & Tiedtke (1984) & Tiedtke (1984) \\
\hline Land surface process & $\begin{array}{l}\text { Simplified simple biosphere scheme } \\
\quad \text { (Xue et al. 1991, 1996; Misra et al. 2002) }\end{array}$ & $\begin{array}{l}\text { Simplified simple biosphere scheme } \\
\quad \text { (Xue et al. 1991, 1996) }\end{array}$ \\
\hline
\end{tabular}

The configuration of the RSM and the COLA AGCM for this study is identical to that in Misra et al. (2003). The RSM is integrated at $80-\mathrm{km}$ horizontal grid resolution with dimensions of 217 (zonal) $\times 112$ (meridional) centered at $15^{\circ} \mathrm{S}, 80^{\circ} \mathrm{W}$; time step of the integration is $240 \mathrm{~s}$. The base field (COLA AGCM forcing) is linearly interpolated in time and bicubic spline interpolated in space to the RSM grid.

\section{Methodology}

In adopting $\mathrm{AN}, \mathrm{MK} 04$ showed that the mean seasonal climate of the South American monsoon improved considerably relative to the conventional way of downscaling. It was particularly reflected in the seasonal mean precipitation, $850-\mathrm{hPa}$ mean circulation field, and intraseasonal variance of the outgoing longwave radiation (OLR). Although a general improvement was seen in the seasonal mean simulation of the South American monsoon from the anomaly nested RSM, it was not clear if anomaly nesting all prognostic variables of the RSM was the most optimal choice. This doubt is also triggered from the fact that NCEP reanalysis suffers from inconsistencies (Kinter et al. 2004; Nigam et al. 2000). Therefore, a set of experiments are designed following the approach of AN (described in MK04) where the AGCM climatology of the nested variables is replaced with the NCEP reanalysis except for one of the nested variables to discern its impact on the simulated climate. In this way five unique experiments (including five ensemble members for each experiment) are defined as given in Table 2. Each of the nested variables is individually isolated: $X$ (translates to the divergent part of the prognostic wind in the RSM; AND), $\zeta$ (translates to the rotational part of the prognostic wind in the RSM; ANV), $T$ (ANT), $\ln \left(p_{s}\right)$ (ANPS), and $q$ (ANQ) in an experiment in which it alone is not bias corrected. On the basis of the results of these experiments the optimal choice of variables for anomaly nesting are isolated over which we then apply the SSBC (in Table 2). In this experiment (EXPT), the time tendencies for a set of chosen bias-corrected (anomaly) nested variables (obtained from the analysis of the results from the previous five experiments) are damped strongly toward the base field tendency for scales exceeding a certain physical length scale (in this study over $1000 \mathrm{~km}$ in wavelength). For the resolution of the NCEP reanalysis of roughly $200 \mathrm{~km}$ and the spatial scale of the interannual anomalies over the continental South America (governed to a large extent by ENSO forcing in the Pacific and the tropical Atlantic Ocean variability) the choice of damping the waves with wavelengths beyond $1000 \mathrm{~km}$ is appropriate. However, the choice of this cutoff wavelength for enforcing the damping of the waves in the RCM is not unique and will be dependent on the domain, the event being simulated, and the forcing data of the RCM. The basis for such a damping scheme may be viewed as analogous to

TABLE 2. Outline of the experiments analyzed in this study.

\begin{tabular}{lcll}
\hline \hline \multicolumn{1}{c}{ Name } & Anomaly nested variable & \multicolumn{1}{c}{ SSBC } & Reference \\
\hline AN-ALL & $\chi, \zeta, \ln p_{s}, T, q$ & Not adopted & Misra and Kanamitsu (2004) \\
ANPS & $x, \zeta, T, q$ & Not adopted & \\
AND & $\zeta, T, q, \ln p_{s}$ & Not adopted & \\
ANV & $x, T, q, \ln p_{s}$ & Not adopted & \\
ANQ & $\zeta, X, T, \ln p_{s}$ & Not adopted & \\
ANT & $\zeta, X, \ln p_{s}, q$ & Adapted to $\zeta$ and $X$ & \\
EXPT & $\zeta, X, \ln p_{s}$ & Not adopted & Misra et al. (2003) \\
CONTROL-B & None & Not adopted & Misra et al. (2003) \\
AGCM & None & & \\
\hline
\end{tabular}


an assimilation scheme in which more weight is given to model analysis (base field tendency) than observations (RSM perturbations derived as deviations of the total tendency from the base field tendency) in regions (at large wavelengths) where observations (RSM perturbations) are unreliable and sparse. The numerical methodology of SSBC is explained in greater detail in appendix B.

In preparing the base field for the RSM anomaly experiments, the AGCM climatology of the nested variable from the AGCM is removed and replaced with the corresponding climatology from observation (in this case the NCEP reanalysis). The construction and replacement of the base field climatology is identical to that described in MK04. It should be noted that all experimental runs were conducted for years that were specifically excluded in creating the climatology.

\section{Design of experiments}

The experiments in this study are conducted for January-March (JFM) of 1997, 1998, and 1999. A total of five ensemble members are run for each year and for each proposed experiment. The control integration (AN-ALL) of this study is the AN run from MK04, which involved anomaly nesting of all prognostic variables of the RSM. The nesting interval is $12 \mathrm{~h}$ for all of the RSM experiments. The initial conditions for all experiments conducted with the RSM in this study are identical to CONTROL-B. For completeness, the COLA AGCM and CONTROL-B seasonal integrations are borrowed from Misra et al. (2003). In CONTROL-B, RSM is nested into the COLA AGCM in the conventional manner. Each ensemble member of the RSM in CONTROL-B was forced at 12-h intervals with the corresponding ensemble member of the COLA AGCM. Unlike any of the AN experiments of this study, no bias correction or damping of any of the waves in the regional model was done in CONTROL-B.

The start and end dates of all the RSM integrations are 0000 UTC 15 December and 1200 UTC 31 March of the following year. The first 15 days of the RSM integration are not analyzed in this study to account for spinup issues.

In all of the model integrations the SST field is updated daily by linearly interpolating from the weekly Reynolds and Smith (1994) optimum interpolation SST dataset. The initial soil moisture fields are obtained from a 2-yr climatology of the Global Soil Wetness Project (Dirmeyer and Zeng 1999). Both of these datasets are linearly interpolated to the RSM grid. An outline of all model integrations used in this study are shown in Table 2.

\section{Results}

In this section we examine the variability at interannual, intraseasonal, and diurnal scales of the various model runs. The interannual variability in JFM seasonal mean precipitation and surface temperature will be verified with Climate Prediction Center (CPC) Merged Analysis Precipitation (CMAP; Xie and Arkin 1996) and Climate Anomaly Monitoring Station (CAMS; Ropelewski et al. 1985) surface temperature, respectively. The intraseasonal variability is examined with OLR for which observational datasets following Liebmann and Smith (1996) are utilized. For a quantitative analysis of the model runs conducted in this study, we have divided our regional domain into smaller subdomains, shown in Fig. 1, for which we will compute the rms error (RMSE), equitable threat score (ETS; a probabilistic skill measure defined in appendix C), and seasonal anomalies where appropriate.

It should be mentioned that the regional model results at $80-\mathrm{km}$ horizontal resolution are verified against large-scale observations that have approximately 300$\mathrm{km}$ resolution. A significant finding of Roads and Chen (2000) was that in comparison to large-scale observations the higher-resolution regional models did not offer any distinct advantage over the more coarsely resolved AGCM. In this study an attempt is made to improve the signal (from the external forcing) in the regional model simulation.

\section{a. Interannual variability}

The JFM seasonal anomalies are computed as the difference from the 3-yr (1997, 1998, and 1999) JFM seasonal mean. Although the number of years is small some of the problems that the models display are endemic and transcend through a number of seasonal integration experiments (120 in total with 40 runs per season). Therefore, differences in the model simulations of the interaannual variability are considered significant. It is important to point out that JFM 1997 was a neutral E1 Niño-Southern Oscillation (ENSO) year [the Niño-3.4 region SST anomaly (SSTA) was $-0.3^{\circ}$ ], 1998 was a strong warm ENSO year (Niño-3.4 region SSTA was $2.0^{\circ}$ ), and 1999 was a relatively weak cold ENSO year (Niño-3.4 region SSTA was $-1.2^{\circ}$ ). It should be noted that, unless specified, all model results in the following discussion are presented from the fivemember ensemble mean.

\section{1) Precipitation}

In Fig. 2 we show the JFM seasonal mean precipitation anomaly from the COLA AGCM, NCEP reanaly- 


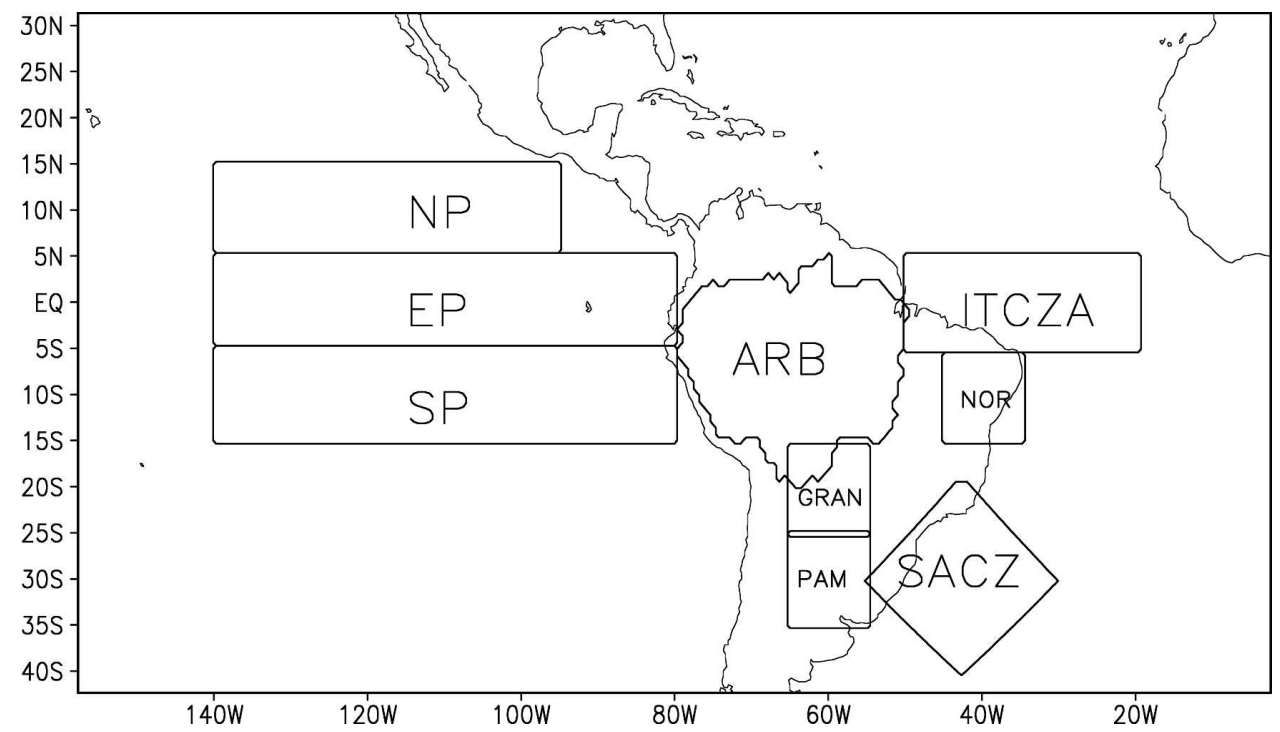

FIG. 1. Outline of the Amazon River basin (ARB), ITCZ over the Atlantic (ITCZA), the Nordeste (NOR), SACZ, Pampas region (PAM), the Gran Chaco area (GRAN), the South Pacific (SP), equatorial Pacific (EP), and North Pacific (NP). (From Misra et al. 2002.)

sis, Control-B and AN-ALL integrations, along with CMAP observations. In relation to CMAP all other model runs, including the reanalysis, show broad agreement over the equatorial Pacific Ocean but show less agreement over the equatorial Atlantic Ocean. In 1998 and 1999 (strong warm and cold ENSO years, respectively) the models are more consistent with observations than in 1997 (neutral ENSO year) over these regions. However, there are apparent differences between the observations and the models, especially over the continental South America, over the subtropical oceans, and just off the equator in both ocean basins. None of the models (excluding the reanalysis) are able to reproduce the observed large anomalies over the Amazon River basin (ARB) or in the subtropical plains of the Pampas (PAM) and Gran Chaco (GRAN) areas (Fig. 1) and over the Nordeste region (NOR) in eastern Brazil. Furthermore, in 1997 the model simulations in these regions verify even more poorly than in 1998 and 1999. The AN-ALL run does show some modest improvements relative to COLA AGCM and CONTROL-B over the ARB, and the equatorial Pacific Ocean. However, it also exacerbates the errors over NOR and over the PAM and GRAN regions, which are also areas of high intraseasonal variance (Paegle and Mo 1997). In many respects, the CONTROL-B integration bears more resemblance to COLA AGCM than to the observations. This can be seen, for example, in the split intertropical convergence zone (ITCZ) in the Pacific Ocean and the absence of anomalies with large spatial extent over the ARB, which was the primary motivation to introduce AN-ALL. But, in terms of seasonal precipitation anomalies the advantage of AN-ALL over the CONTROL-B runs is somewhat minimal.

To provide a more quantitative analysis, we have plotted the RMSE in Fig. 3 for all six AN nested runs including AN-ALL. It is clearly seen from this figure that in the tropical regions of ARB, the ITCZ over the Atlantic (ITCZA), and the South Pacific (SP) the RMSE in ANQ and ANT are relatively small [with the equatorial Pacific (EP) and North Pacific (NP) being the exceptions]. However, a more significant result that can be seen from this figure is that the RMSE of ANALL is the largest in most regions, suggesting that bias correction to all the prognostic variables of the RSM may not be the most optimal strategy for reducing precipitation errors. Similarly, we have plotted the ETS for a relatively easy but a relevant threshold of positive and negative precipitation anomalies for the regions shown in Fig. 4. We notice in most regions the threat scores of ANQ and ANT experiments are relatively high.

We have plotted the 200-hPa temperature anomalies in Fig. 5 for all of the AN experiments and NCEP reanalysis. It is consistently seen from the figure that in all three years the temperature anomalies from the ANQ, ANT, and AN-ALL experiments match relatively closely with the NCEP reanalysis anomalies. However, in ANPS, AND, and ANV experiments there is evidence of some deterioration in the simulation. The results in Figs. 3, 4 and 5 suggest that anomaly nesting of $T$ and $q$ variables may be overconstraining the RSM 

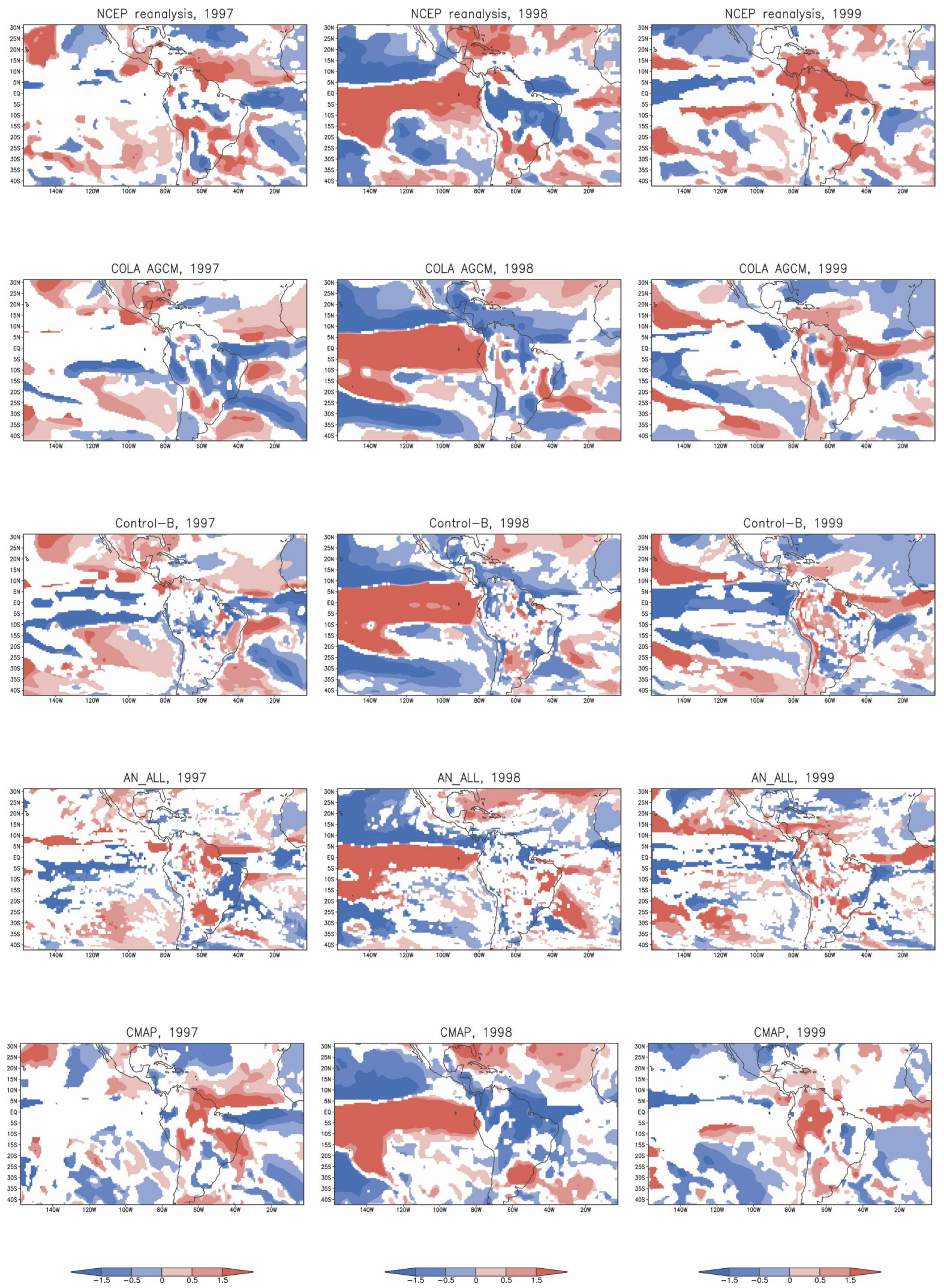

FIG. 2. JFM seasonal mean precipitation anomalies $\left(\mathrm{mm}^{\text {day }}{ }^{-1}\right)$. Only statistically significant anomalies according to a Student's $t$ test are shaded. 


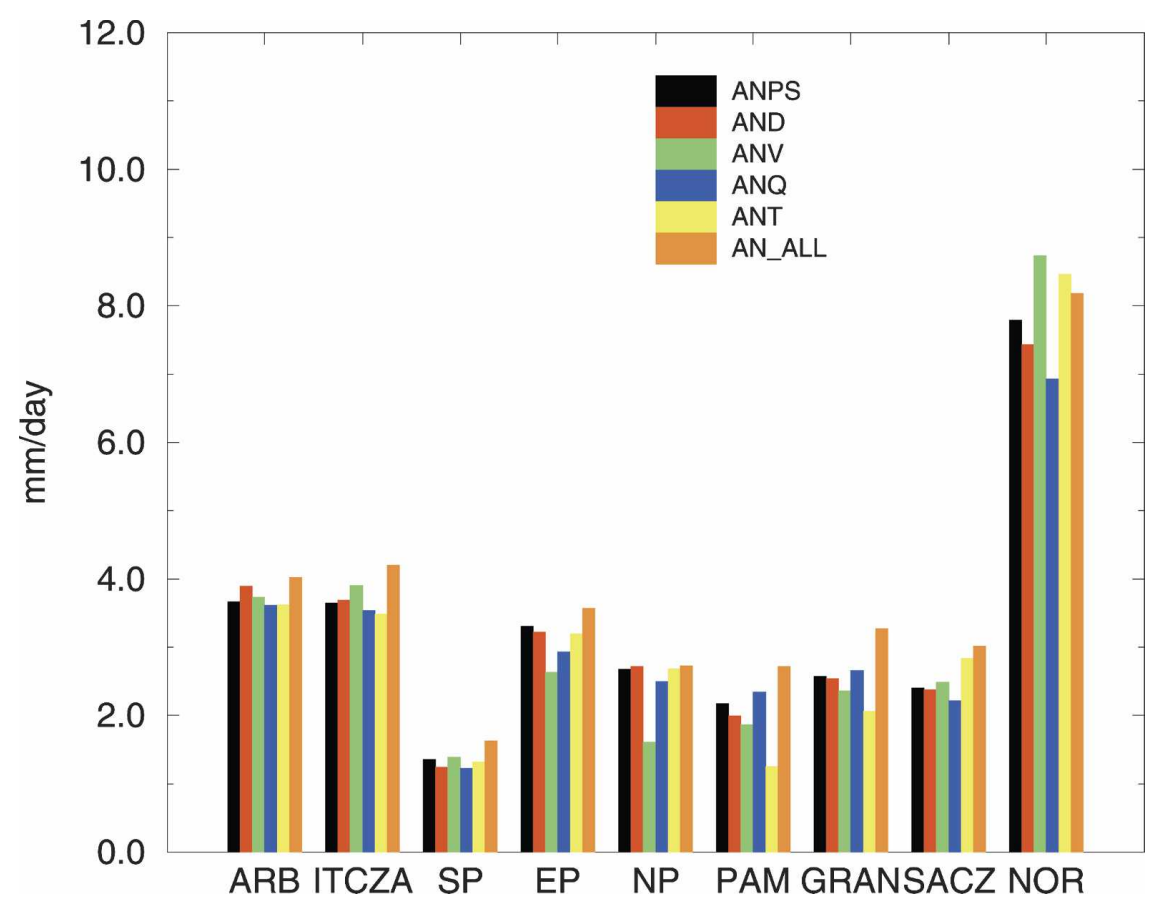

FIG. 3. The rms error of the JFM seasonal precipitation anomalies over the regions shown in Fig. 1 from the anomaly nested runs ANPS, AND, ANV, ANQ, ANT, and AN-ALL.
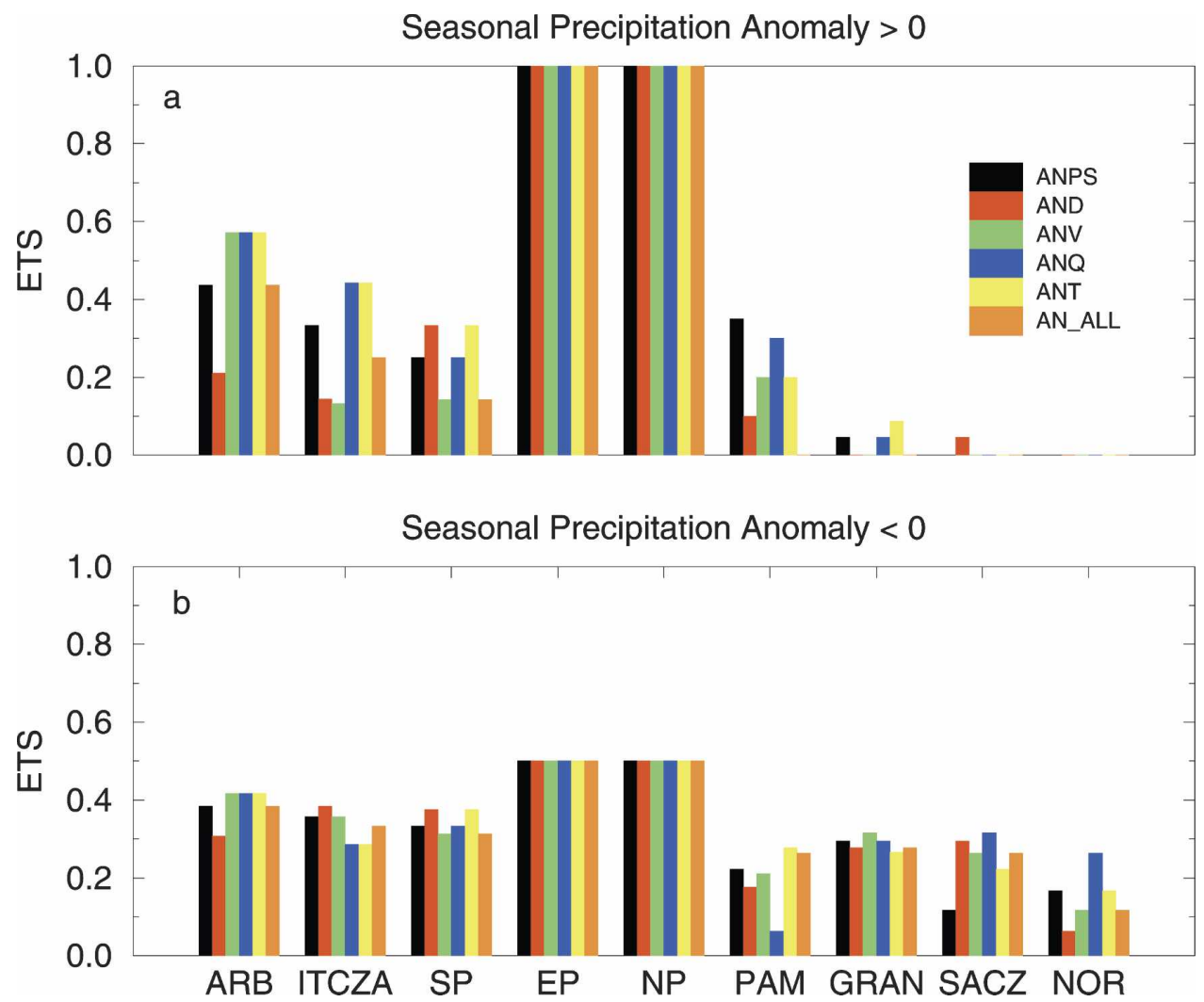

FIG. 4. The equitable threat score of the JFM precipitation anomalies over the regions shown in Fig. 1 from the anomaly-nested runs ANPS, AND, ANV, ANQ, ANT, and AN-ALL. 

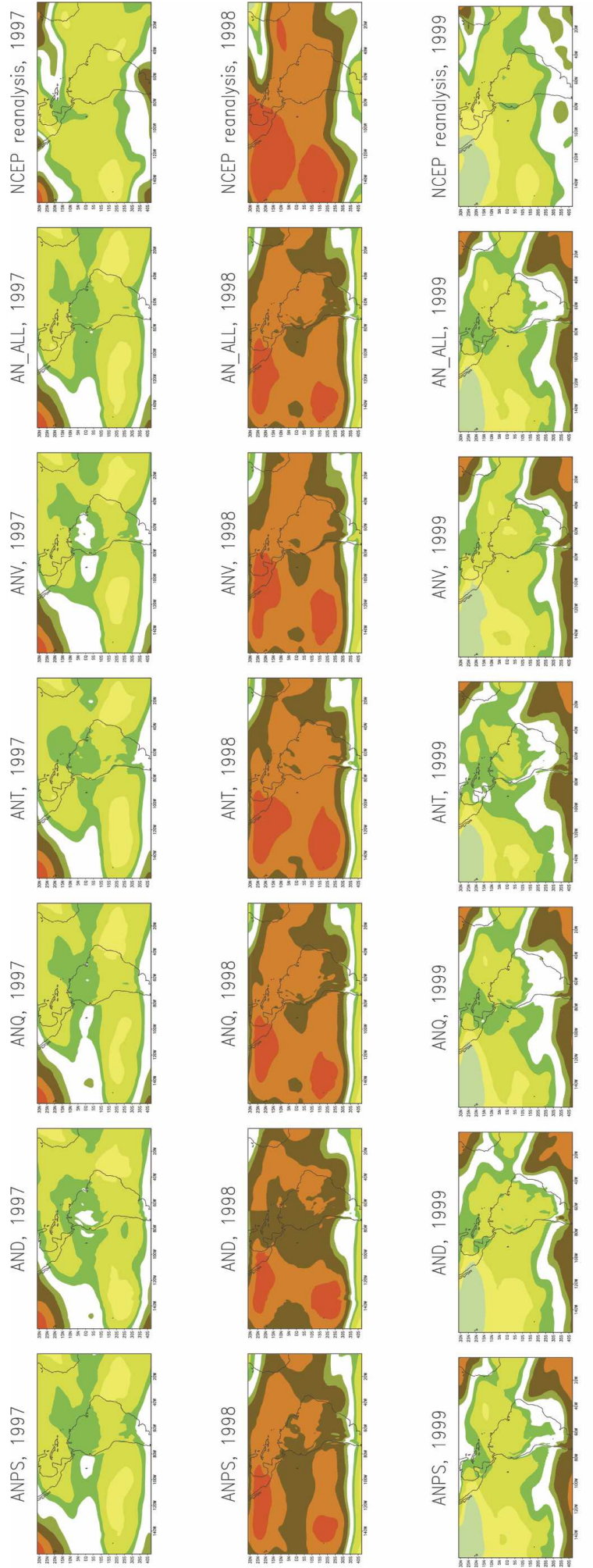

FIG. 5. JFM seasonal mean 200-hPa temperature anomalies from the six anomaly nesting experiments ANPS, AND, ANQ, ANT ANV, AN-ALL, and NCEP reanalysis. simulations. This result is consistent from our earlier study (MK04), which pointed out that the convection scheme in the RSM had to be made similar to that of the COLA AGCM (relaxed Arakawa-Schubert scheme; Moorthi and Suarez 1992) to reduce the huge precipitation bias in the AN-ALL integration. Furthermore, owing to the paucity of observations and the importance of convection in the Tropics, the resulting analysis of especially $T$ and $q$ is strongly influenced by the convective scheme of the assimilating model. Therefore, in the approach of using SSBC and AN, differing physics, dynamics, and resolution between the reanalysis and the driving model (in our case COLA AGCM) has to be reconciled while forcing the RSM. This also suggests that the climatology of the RSM over this domain is strongly influenced by the convection scheme.

These results from AN integrations provided the background knowledge to design the subsequent experiment that involved employing the SSBC. In this run the bias correction (AN) to the base field of $T$ and $q$ was avoided, while it was applied to the rest of the fields. The damping of the larger scales resolved by the RSM (SSBC) is applied only to the anomaly nested zonal and meridional components of the wind with the tacit assumption that the large-scale errors in $T$ and $q$ will also be reduced through nonlinear interactions. Similarly, in the EXPT run the natural logarithm of surface pressure $\left[\ln \left(p_{s}\right)\right]$ perturbation is set to the mean difference of the surface pressure elevation between the coarse surface elevation of the COLA AGCM and the regional finescale surface elevation of the RSM. This is meant to correct the anomalous pressure difference that is inadvertently introduced through the use of separate orography in the AGCM and the RSM. It may be noted that the technique of SSBC adopted in this study diverges from the one adopted in KK07 in at least three different ways. One, this is being adapted to the RSM when it is forced with bias-corrected AGCM forcing rather than the reanalysis. Second, the SSBC is done only to the rotational and divergent component of the winds and the natural log of surface pressure. The variables of $T$ and $q$ are untouched. The damping coefficient is almost twice as large compared to that used in KK07.

The seasonal precipitation anomalies as shown in Fig. 2 are now shown from the EXPT integration in Fig. 6. There is a marked improvement in the seasonal anomalies over most regions including the tropical $\mathrm{Pa}$ cific Ocean and the ARB. However, over the subtropical plains of PAM and GRAN, the South Atlantic convergence zone (SACZ), and the NOR the results are more sobering. The EXPT is unable to rectify the 

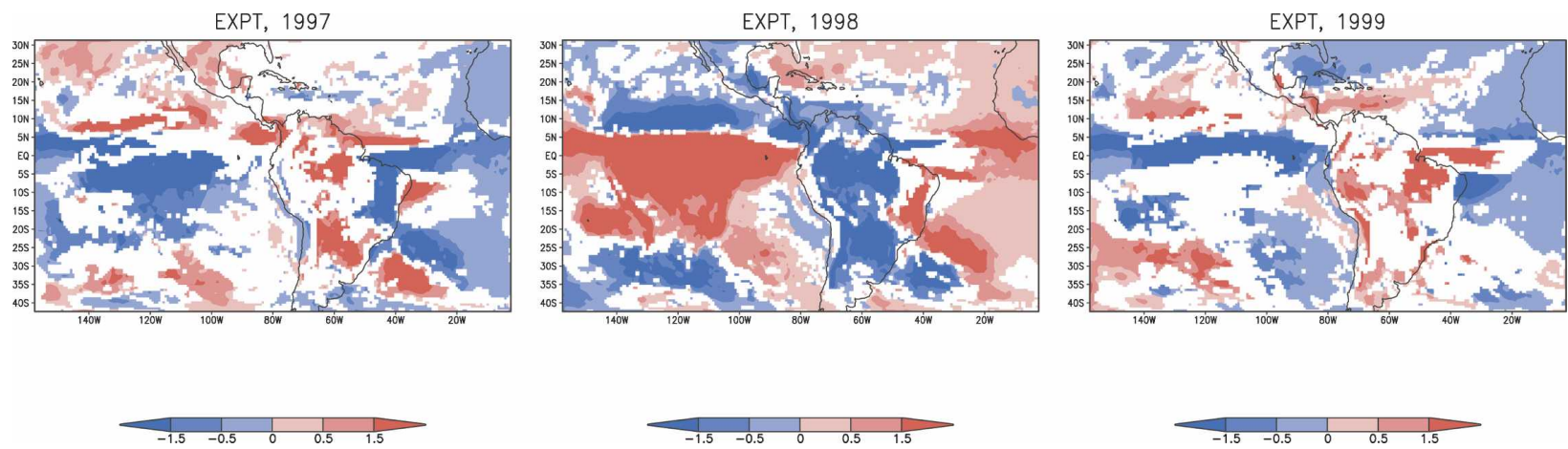

FIG. 6. As in Fig. 2 but from the EXPT integration.

prevalent erroneous interannual seasonal anomalies displayed by the AN-ALL and subsequent AN experiments (not shown) over these regions. It should however be noted that the methodology adopted in EXPT critically depends on the quality of the NCEP reanalysis. Some of the features such as the precipitation anomalies over the tropical Atlantic Ocean and off the equator in the Pacific Ocean in the EXPT are comparatively more similar to the NCEP reanalysis than other
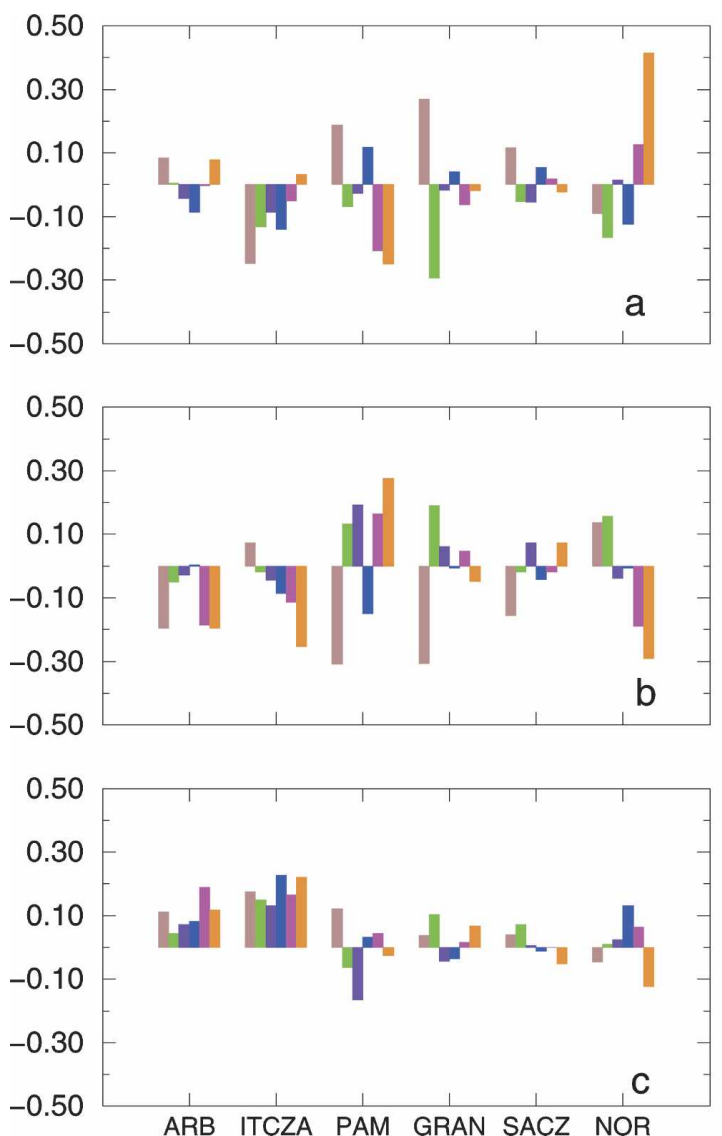

RSM runs of this study. The efficacy of the adopted methodology in EXPT will therefore benefit from improvements in the reanalysis.

In Fig. 7 we have plotted the mean JFM seasonal precipitation anomalies for the EXPT, AN-ALL, CONTROL-B, COLA AGCM, and NCEP reanalysis, and the CMAP observations for the domains shown in Fig. 1. We have plotted the precipitation anomalies for the NP, EP, and SP regions separately because of their
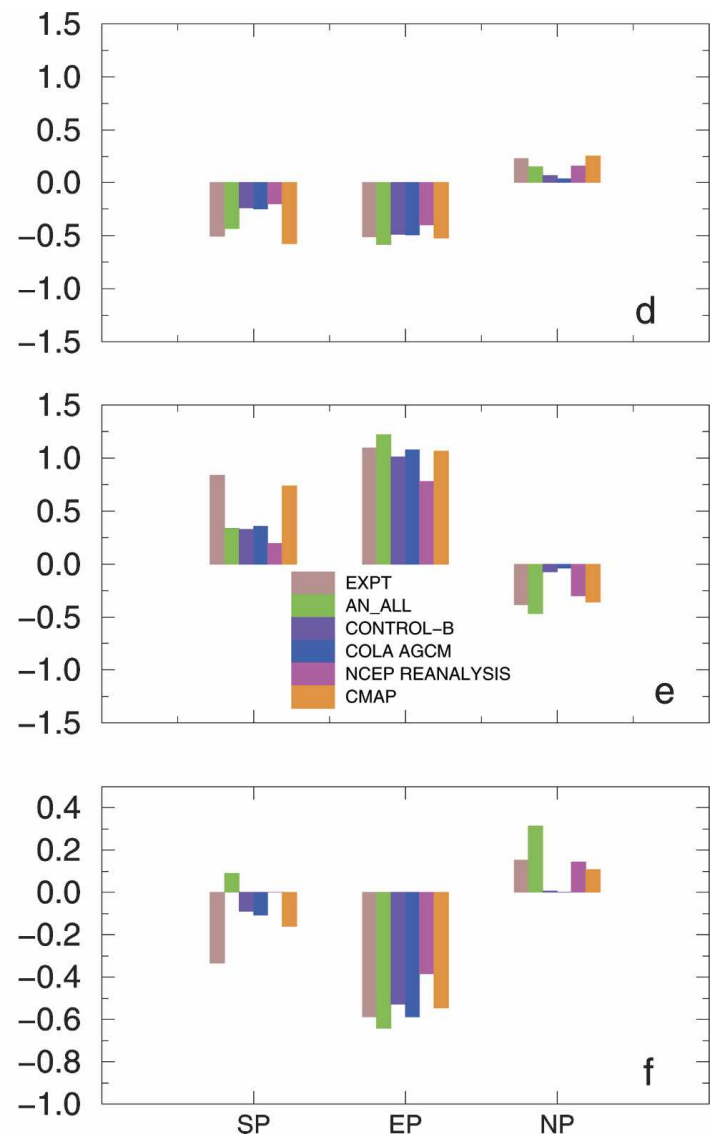

FIG. 7. The mean JFM seasonal precipitation anomalies averaged over the regions shown in Fig. 1 for (a), (d) 1997, (b), (c) 1998, and (c), (f) 1999. 


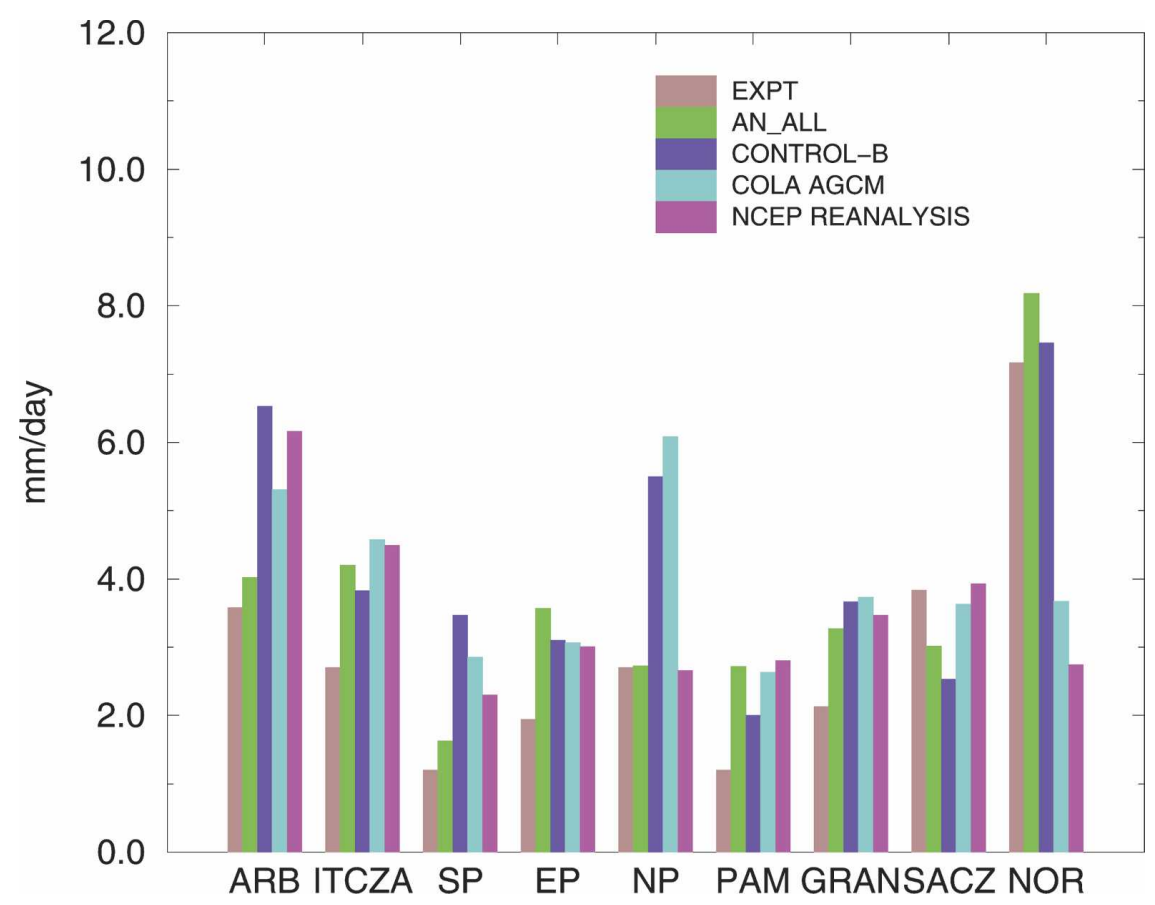

FIG. 8. As in Fig. 3 but for EXPT, AN-ALL, CONTROL-B, COLA AGCM, and NCEP reanalysis.

larger anomalies relative to other regions delineated in Fig. 1. Over the tropical regions of ARB, ITCZA, SP, $\mathrm{EP}$, and NP the anomalies from EXPT are relatively closest to the observations consistently in all three years. In the subtropical PAM, SACZ, and GRAN regions and in the NOR the results are not so encouraging from the EXPT. The RMSE of these model runs are shown in a similar format in Fig. 8. This figure corroborates the conclusions from Fig. 7 that over the Tropics EXPT shows a superiority in precipitation simulation over other model runs, while over the subtropical regions of SACZ and the NOR in eastern Brazil the results are not as encouraging. The ETS calculated for the thresholds of positive and negative precipitation anomalies is shown in Fig. 9, which indicates that the precipitation simulation over the ARB, ITCZA, SP, $\mathrm{EP}$, and NP regions benefit from the methodology adopted in EXPT.

In Fig. 10 we have plotted the $200-\mathrm{hPa}$ temperature anomalies from the EXPT and CONTROL-B runs. In comparison with Fig. 5 and CONTROL-B (in Fig. 10), EXPT has improved the simulation of the temperature anomalies in all three years.

\section{2) Surface temperature}

The JFM seasonal anomalies of surface temperature are shown in Fig. 11 in a format similar to that in
Fig. 2. The model runs represented by COLA AGCM, CONTROL-B, and AN-ALL are able to simulate the gross large-scale patterns such as the relatively warm (cold) land surface over the ARB in 1998 (1999). However, there are also apparent differences between the models. It is apparent from the figure that the COLA AGCM simulations have some of the largest errors such as the absence of anomalies over the ARB in 1997 and in the subtropical region of PAM in all three years. The CONTROL-B RSM runs, which were nested into the COLA AGCM simulations, did improve upon the large-scale model in areas over and around eastern Brazil. But, it also introduced erroneous anomalies in the PAM area in 1998 and did not improve the simulation over the ARB region. The AN-ALL run made significant improvements over the ARB and the PAM regions, thus supporting the notion that improvement in the large-scale mean state of the atmosphere has a bearing on the simulation of the land state variables in the RSM. However, despite these improvements in the AN-ALL integration there are significant differences from the observations, especially in the anomalies over the PAM region. It should be mentioned that the NCEP reanalysis represents the observed seasonal anomalies fairly well relative to the rest of the model runs. The other AN experiments showed close resemblance to the AN-ALL integration (not shown), suggesting that it is not possible to isolate systematic errors 

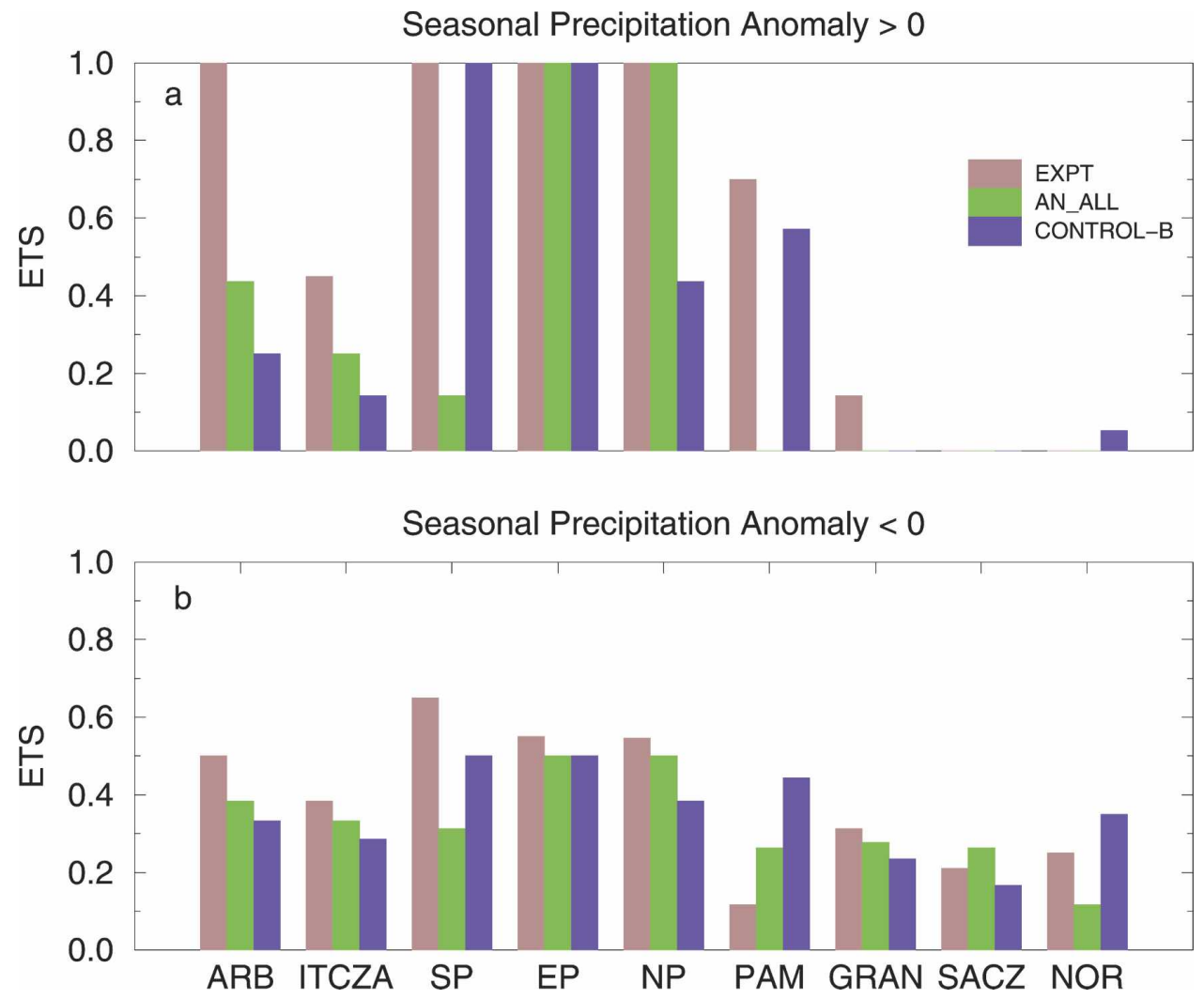

Fig. 9. As in Fig. 4 but for EXPT, AN-ALL, CONTROL-B, and COLA AGCM.

CONTROL-B, 1997

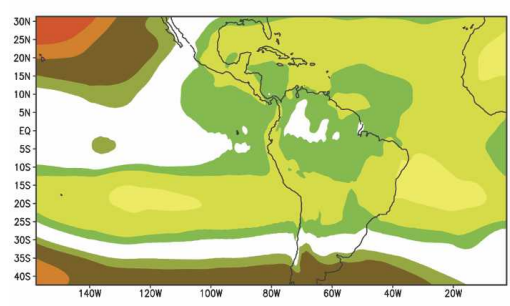

EXPT, 1997
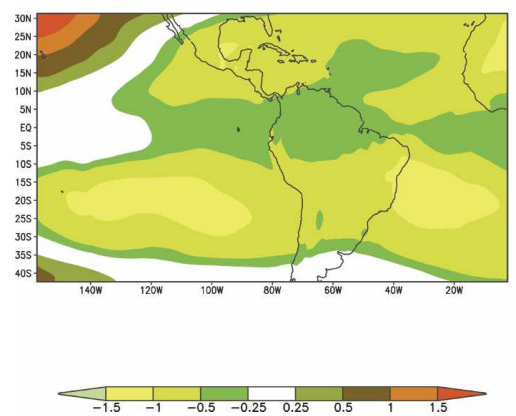

CONTROL-B, 1998

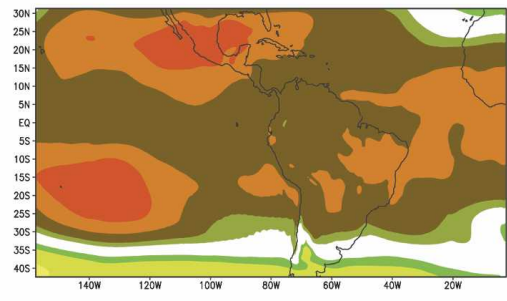

EXPT, 1998
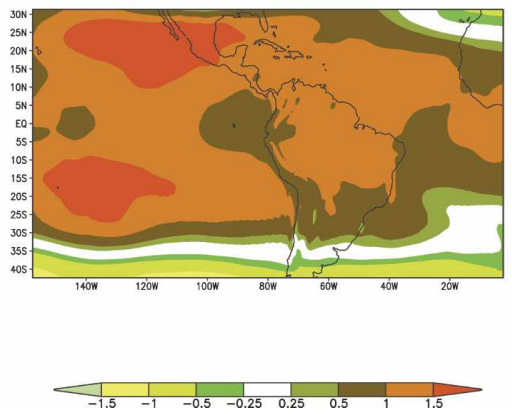

CONTROL-B, 1999

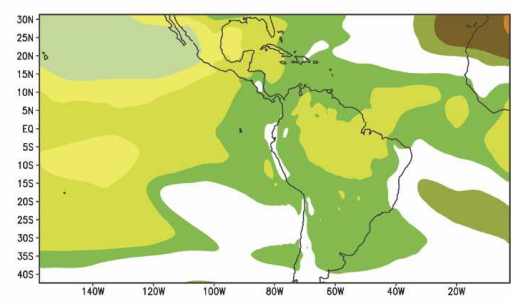

EXPT, 1999
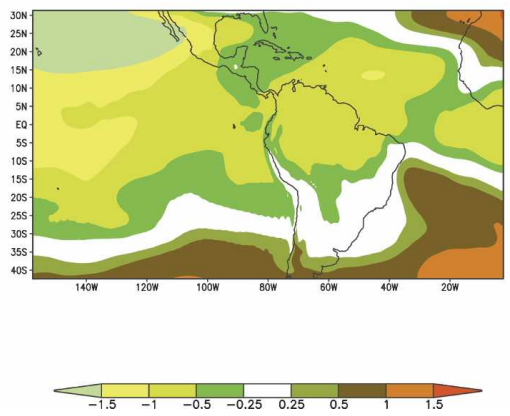

FIG. 10. As in Fig. 5 but for EXPT and CONTROL-B. 

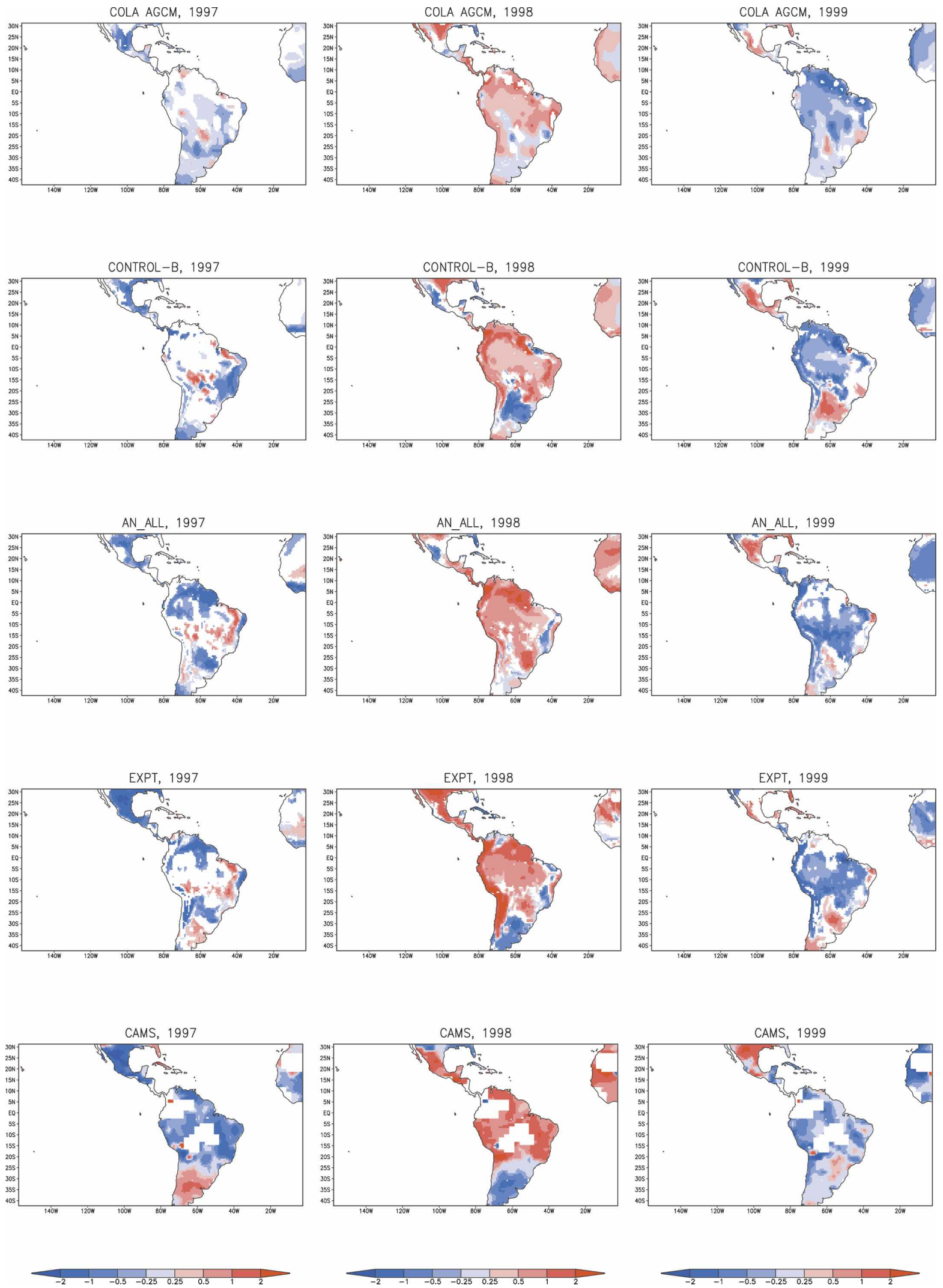

FIG. 11. As in Fig. 2 but for surface temperature anomalies. The unshaded regions over land in the observations (CAMS) denote missing data. Only statistically significant values according to a Student's $t$ test are shaded. 
in the base field of a single atmospheric variable to explain its influence on the RSM land surface state variable simulation. The EXPT run in Fig. 11 clearly shows significant improvements over the rest of the model runs, especially in the PAM region and in central America, without deteriorating any of the verifiable results of the AN-ALL integration. This result suggests that addressing the issue of systematic errors in both the RCM and in the forcing field is beneficial in simulating the seasonal anomalies of the land state variables.

\section{b. Intraseasonal variability}

In MK04 and Misra (2004) it was clearly demonstrated that AN showed reasonable agreement with observations in terms of the JFM seasonal mean intraseasonal (20-40 days) variance of OLR. It was further shown that this improvement in anomaly nested RSM integrations over the COLA AGCM and the CONTROL-B runs was primarily due to a diurnal rectification of the base field tendency imposed by the NCEP reanalysis climatology. The variance of filtered OLR on 20-40-day time scales is shown in Fig. 12. The AN-ALL runs have a relatively vigorous intraseasonal activity over the eastern Pacific Ocean in 1998 and over the SACZ and eastern Brazil region in all three years. In the EXPT run (Fig. 12) the intraseasonal variance is significantly reduced relative to AN-ALL over these regions bringing it closer to the observations. This reduction in the intraseasonal variance in the EXPT integration is primarily from the SSBC, as all of the AN experiments showed intraseasonal variance comparable to that in AN-ALL run (not shown).

\section{c. Diurnal variability}

The reduction in the intraseasonal variance of OLR over eastern Brazil and the SACZ region in the EXPT run occurs in part due to the modulation of the diurnal variability of precipitation relative to the AN-ALL run in some of these intraseasonally active regions. In Fig. 13 we display both the JFM seasonal mean diurnal precipitation range (DPR) determined from 6-hourly model output from the EXPT integration and its deviation from the other RSM runs. It is seen that over the western ARB (eastern Brazil and SACZ) the DPR has increased (decreased) in the EXPT relative to ANALL. CONTROL-B shows the largest DPR of all the other RSM runs in Fig. 13. The COLA AGCM tends to have large diurnal amplitudes even over open oceans, which when imposed on the RSM results in reducing the intraseasonal variability (MK04). The five AN ex- periments show very little difference between them, suggesting that the diurnal rectification in $\mathrm{AN}$ is not imposed by bias correction in the base field of any one prognostic variable of the RSM.

\section{Discussion}

Regional modeling is also perceived as a kind of data assimilation (von Storch et al. 2000). This analogy follows from the similarity in prescribing the large scale from the lateral boundary condition in a regional model to using relatively coarsely spaced station observations to produce gridded analysis at comparatively finer resolution in a data assimilation scheme. So, if we were to use this analogy and make an attempt to interpret the results of this study, then it may be said that the use of bias-corrected large-scale (base field) thermodynamic variables of $T$ and $q$ are inappropriate, just as coarsely spaced, unreliable observed soundings can sometimes inappropriately describe the large scale, and therefore have to be discarded by the quality control check of the data assimilation. As a consequence, SSBC cannot be applied to the $T$ and $q$ variables. The rejection of bias correction to $T$ and $q$ in this methodology primarily stems from the difference in the physics and resolution of the driver model, reanalysis, and the RSM. In a region such as the deep Tropics there is a definite paucity of observations as a result of which model bias has relatively larger influence on the analysis. These ( $T$ and $q$ ) variables thus include the systematic errors of the AGCM. On the other hand, the bias-corrected large-scale vorticity, divergence, and $\ln p_{s}$ serve as a more reliable substitute than the full AGCM values. This is akin to using reliable and more densely available wind and surface station observations instead of the first-guess field from the model short-term forecasts in a data assimilation scheme. As a result, the large scale described by such a corrected base field is given more weight than the AGCM values by the SSBC. That these analogies to data assimilation are not overreaching becomes clear when considering the distribution of surface station, radiosonde, pilot balloon, aircraft reports, drifting buoys, and dropwinsondes (Daley 1991) that are more dense over land regions in the midlatitudes than over the open tropical oceans (Daley 1991).

Recently, Bengtsson et al. (2004) showed that the assimilation of satellite-based humidity observations introduced errors in the assimilation that resulted in an erroneous global water budget. They argue that it is unnecessary to assimilate $q$ since it can be generated by a "skillful" model in a manner consistent with the associated dynamics and surface fluxes. Likewise, it may be argued here that imposing bias-corrected forcing of 

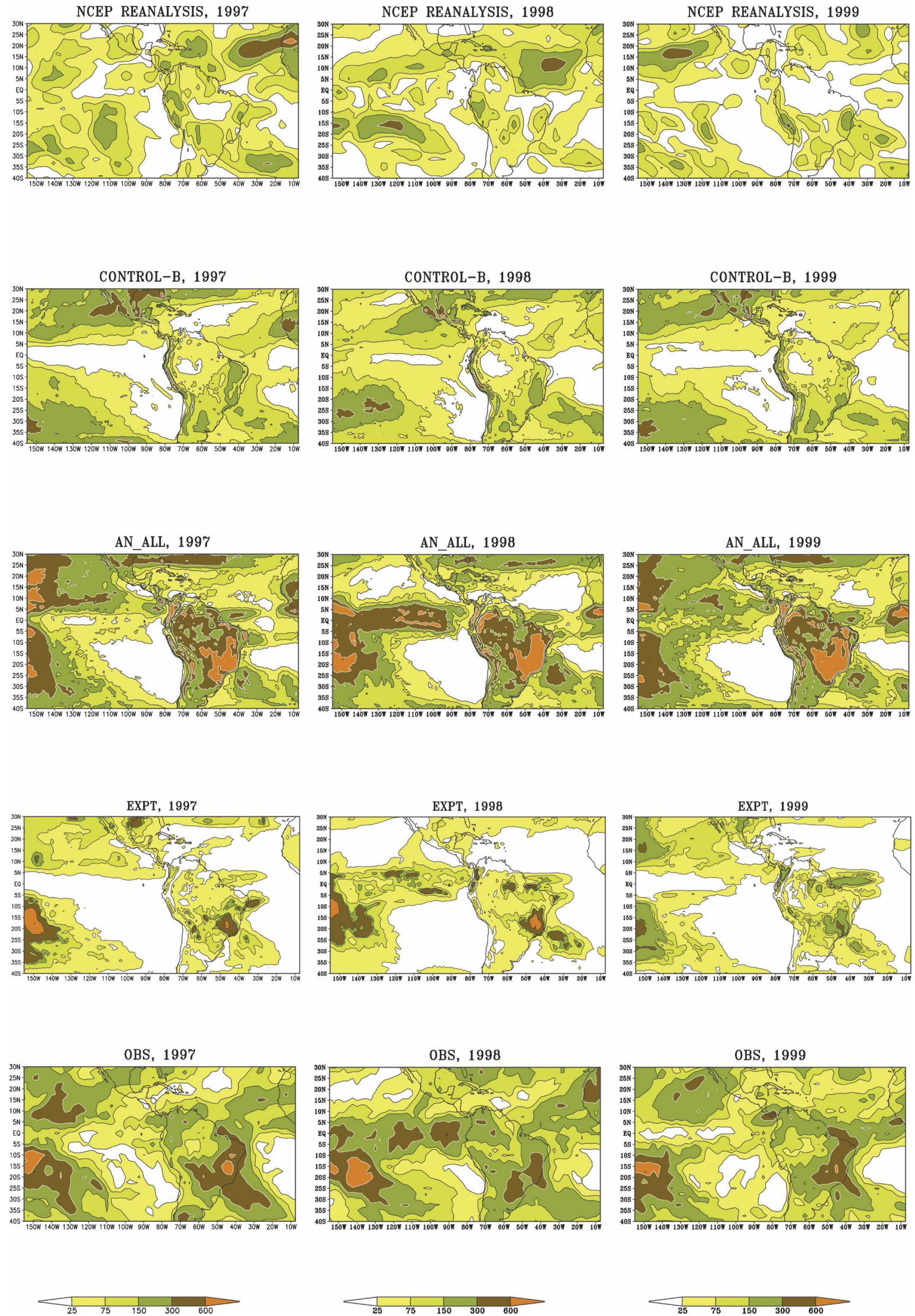

FIG. 12. The variance of the filtered OLR anomalies at 20-40-day time scale for the JFM season of 1997, 1998, and $1999\left(\mathrm{~W}^{2} \mathrm{~m}^{-4}\right)$. 

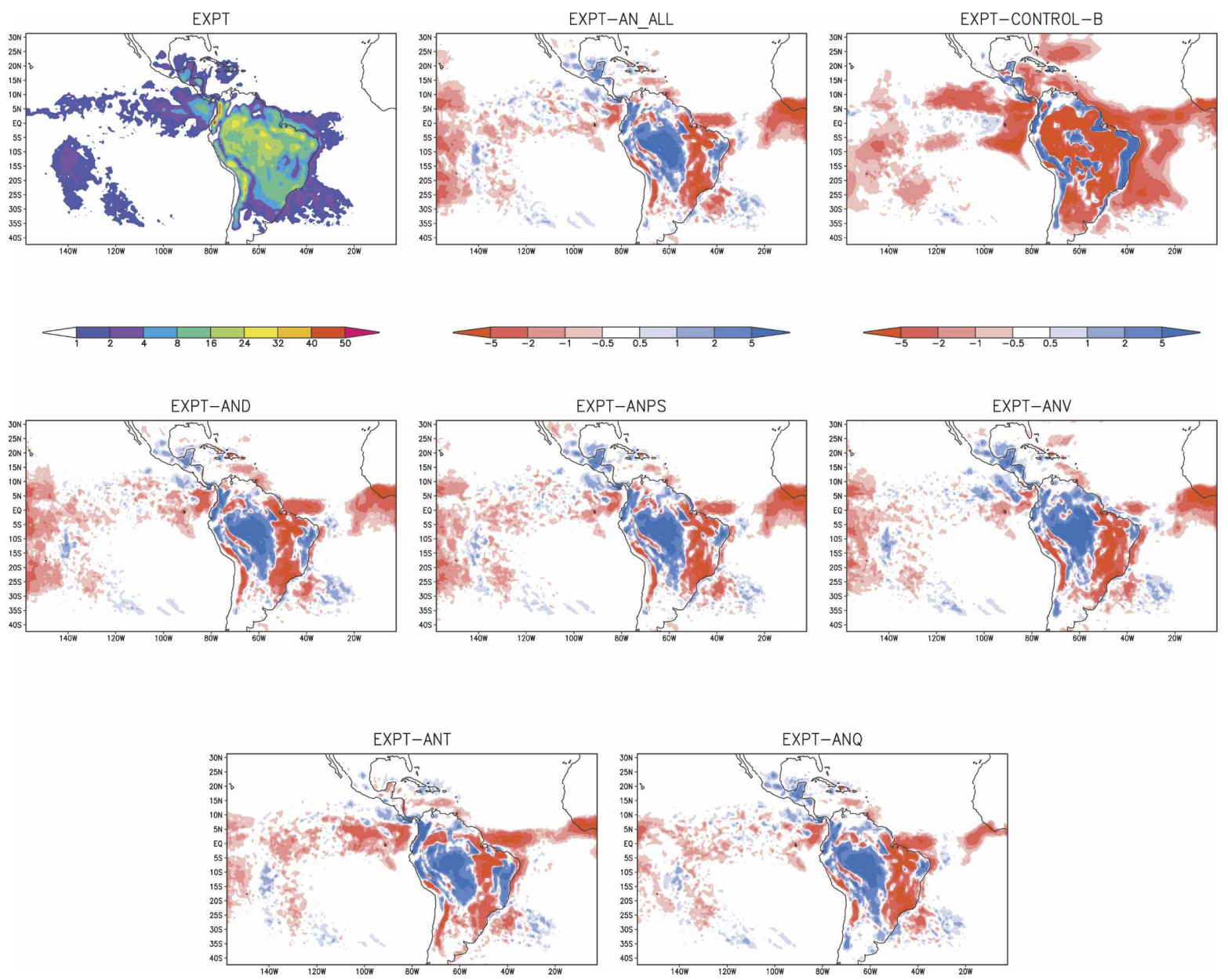

FIG. 13. The mean JFM diurnal precipitation range from EXPT and its difference from the rest of the model runs $\left(\mathrm{mm} \mathrm{day}^{-1}\right)$.

the thermodynamic variables to RSM may be unnecessary.

Though regional modeling and data assimilation have some broad similarities, they are not perfect analogies either. The base field in the RCM is prescribed at regular time intervals on a regular grid, unlike the observations in a data assimilation scheme, which can be asynoptic. Furthermore, the base fields of all variables being prescribed came from a single (consistent) AGCM, unlike observations that can come from a variety of platforms. The fact that using the uncorrected RCM predicted fields of $T$ and $q$ in the presence of the large-scale corrections of the RCM predicted $u, v$, and $\ln p_{s}$ is successful suggests that largescale dynamics has a positive influence on the thermodynamics in the evolving regional climate over South America and surrounding ocean basins.

On a more philosophical note, the parameterization processes in the numerical models can be conceptualized as a downscaling process. Parameterization schemes are founded on the basis that the subgrid-scale processes can be represented in terms of the large-scale forcing, a rationale similar to that for the use of regional models. Therefore, it is possible to draw this analogy to the improvement of seasonal convective precipitation anomaly over the ARB and the surface temperature anomalies over the subtropical South America in the EXPT run. In the set of EXPT integrations, through SSBC and AN we have improved the large-scale forcing that in turn simulates the parameterized processes of convection and land surface better.

In a related study Wang et al. (1999), using a variable resolution model, showed that the degree of imposition of divergent flow and thermal field has a significant bearing on the simulation of the precipitation on the high-resolution grid.

\section{Conclusions}

In this study an attempt has been made to further improve on the regional climate simulation of the 
SASM from our past studies (MK04; Misra et al. 2003). The basic tenet of the proposed methodology is to force the regional model with the best estimate of the large scale other than the reanalysis and restrict the growth of perturbations in the RCM. This is achieved by first identifying the two main sources of systematic errors in the RCM, namely, the prescribed lateral boundary conditions from an AGCM to the RCM and the deficiencies of the RCM itself. The former is addressed by removing the climatology of some of the selected nested variables of the AGCM and the latter is partially addressed by constraining the solution of a set of preselected prognostic variables of the RCM at the largest regional scales to what is prescribed from the base field. The selection of the nesting variables subject to corrections in the climatology of its base field and the restriction of its evolution by the SSBC are carried out after conducting a host of sensitivity experiments. The SSBC proposed here diverges from the seminal work of KK07 on this subject in three ways. One, we have adapted this methodology to RSM forced with bias-corrected largescale forcing from an AGCM. Second, we impose SSBC only on rotational and divergent components of the wind along with the natural log of surface pressure. Third, the damping coefficient is much larger (almost twice as large) relative to KK07. These experiments showed that, if the $T$ and $q$ variables are left to evolve in the RSM with all of the degrees of freedom while the dynamic fields of $u, v$, and $\ln p_{s}$ are corrected through AN and SSBC, a superior simulation of the SASM is achieved compared to earlier results (see MK04; Misra et al. 2003 and various sensitivity experiments conducted in this study especially in tropical regions).

Although we have provided some "a posteriori" physical reasoning on the choice of the nested variables that have been selected for bias correction and subsequently subjected to SSBC by drawing an analogy to a data assimilation scheme, the approach adopted here is basically empirical. It is difficult to conceive "a priori" the most sensitive nesting variables in a RCM without conducting such sensitivity experiments. The sensitivity of the RCM simulations is multifaceted. It is a function of domain size and location, model (RCM and AGCM), nesting interval, and season. This forces such an empirical approach to regional modeling.

This methodology also extends the potential use of reanalysis in climate prediction. Therefore, an improved reanalysis borne from better observational coverage and use of better models would not only yield improvements in the climate diagnostic information but also has the potential to substantially improve anomaly nested regional prediction models.
Acknowledgments. The author was inspired by the seminal work of Drs. Hideki Kanamaru and Masao Kanamitsu on this subject of SSBC. I am greatly indebted to Kanamitsu for his guidance through the course of this work and his humility to be acknowledged and not made as coauthor of this paper. However, I must confess that shortcomings in this paper if any are entirely mine. The author would like to thank Drs. B. P. Kirtman and David Straus for making useful comments on an earlier version of the manuscript. The author also thanks the useful review comments of an anonymous reviewer. The author would also like to acknowledge the use of interpolated OLR obtained from the NOAA-CIRES Climate Diagnostics Center, in Boulder, Colorado, from their Web site at http:// www.cdc.noaa.gov/. This study was supported by NSF Grant ATM9814295, NASA Grant NAG5-11656, and NOAA Grant NA16GP2248.

\section{APPENDIX A}

\section{Step-by-Step Computational Procedure in the RSM}

1) At any given time step, the regional model perturbation can be calculated as

$$
A_{r}(x, y)=A_{t}(x, y)-A_{g}(x, y),
$$

where $A_{g}$ is the AGCM field or alternatively the base field, and $A_{t}$ is the analysis over the regional domain. At initial time $A_{t}$ will be nearly identical to $A_{g}$ in the absence of a independent analysis at the RSM resolution and domain; $A_{t}$ is the total field predicted by the regional model; $x, y$ are the RSM grid coordinates.

2) A wall boundary condition is then imposed on $A_{r}(k$, $l$ ) obtained from Fourier transforming $A_{r}(x, y)$, where $k$ and $l$ are the zonal and meridional wavenumbers in Fourier harmonics.

3) The required spatial derivates of $A_{r}$ are evaluated spectrally.

4) Similarly $A_{g}(m, n)$ is obtained from $A_{g}(x, y)$ using spherical harmonics and the required spatial derivates are computed; $m$ and $n$ are zonal and meridional wavenumbers in spherical harmonics.

5) The spatial derivates of the total field $A_{t}(x, y)$ are then computed in grid space by adding the spatial derivatives evaluated in the previous steps.

6) The full-model tendencies are then calculated $\left(\partial A_{t}(x, y) / \partial t\right)$. Note that this tendency is nonzero at the boundaries.

7) The perturbation tendency is computed now as 


$$
\frac{\partial A_{r}(x, y)}{\partial t}=\frac{\partial A_{t}(x, y)}{\partial t}-\frac{\partial A_{g}(x, y)}{\partial t} .
$$

The wall boundary condition is imposed on $\partial A_{r}(k$, l) $/ \partial t$.

8) Then $A_{r}(k, l)$ is advanced in time as

$$
A_{r}(k, l)_{t+\delta t}=A_{r}(k, l)_{t-\delta t}+2 \delta t \frac{\partial A_{r}(k, l)}{\partial t} .
$$

The results are Fourier transformed to $A_{r}(x, y)_{(t+\delta t)}$ and returned to step 3 .

\section{APPENDIX B}

\section{The Spectral Damping Scheme in the RSM}

In the RSM, although the global field is used in the entire domain (not only at the boundary), it is only applied to reduce the error in the regional domain-scale calculations. Since in the control RSM there is no explicit forcing toward the global model field, the perturbation can grow to a large amplitude and eventually modify the domain-scale motion. To reduce this bias, the spectral damping scheme is applied in the following manner:

$$
\frac{\partial A_{t}}{\partial t}=\left[\frac{\partial A_{r}}{\partial t}+\eta \frac{\partial A_{g}}{\partial t}\right] /[1+\eta],
$$

where $\eta$ is the damping coefficient and rest of the notations follow from the previousappendix; this damping scheme is applied after the perturbation is computed in step 7 of appendix A.

In the above equation, setting $\eta$ to zero will yield the control model. If this scheme is adopted at all spatial scales, then in the limit that $\eta$ tends to a large finite number the use of the RSM becomes redundant. Since the NCEP reanalysis is imperfect due to model bias, deficit of observations, bias of the assimilation scheme, observational errors, and the RSM nonlinear scale interactions that may also lead to errors, attaining an optimal choice of the damping coefficient is difficult. Another apparent limitation of this scheme is that the global tendency term in Eq. (B1) is computed at every time step from linearly interpolating the tendency calculated at the nesting interval. In other words, this scheme could be sensitive to the nesting interval in regions and/or seasons where the short-term variability is large. Taking into consideration all of these factors and after conducting some experimental integrations, we arrived at a choice of $\eta=2.0$ as an optimal choice for this study.

The perturbation tendencies are then recomputed with these new damped total fields (step 7 of appen-
TABLE C1. Contingency table for calculating equitable threat score.

\begin{tabular}{cll}
\hline \hline \multirow{2}{*}{$\begin{array}{c}\text { Is the } \\
\text { event observed? }\end{array}$} & \multicolumn{2}{c}{ Model forecast } \\
\cline { 2 - 3 } Yes & Hit $(H)$ & \multicolumn{1}{c}{ No } \\
\hline No & False alarm $($ FA) & $\begin{array}{l}\text { Miss }(M) \\
\text { Correct rejection }(\mathrm{CR})\end{array}$ \\
\hline
\end{tabular}

dix A). The wall boundary condition is imposed on $\partial A_{r}(k, l) / \partial t$. and there upon advanced in time as in step 8 of appendix A. It should be noted that the above spectral damping scheme in this study is employed only on the wind components and $\ln p_{s}$ fields.

\section{APPENDIX C}

\section{Equitable Threat Score}

The equitable threat score (ETS) measures the fraction of observed and/or forecast events that were correctly predicted, adjusted for hits associated with random chance. Thus, given a contingency table (shown in Table C1), the equitable threat score (ETS) can then be mathematically written as

$$
\mathrm{ETS}=\frac{\left(H-H_{\text {random }}\right)}{H+M+\mathrm{FA}-H_{\text {random }}},
$$

where

$$
H_{\text {random }}=\frac{(H+M)(H+\mathrm{FA})}{(H+M+\mathrm{FA}+\mathrm{CR})} .
$$

\section{REFERENCES}

Antic, S., R. Laprise, B. Denis, and R. de Elia, 2004: Testing the downscaling ability of a one-way nested regional climate model in regions of complex topography. Climate Dyn., 23, 473-493.

Bengtsson, L., K. I. Hodges, and S. Hagemann, 2004: Sensitivity of large-scale atmospheric analyses to humidity observations and its impact on the global water cycle and tropical and extratropical weather systems in ERA40. Tellus, 56A, 202217.

Chou, M.-D., 1992: A solar radiation model for use in climate studies. J. Atmos. Sci., 49, 762-772.

Christensen, O. B., J. H. Christensen, B. Machenhauer, and M. Botzet, 1998: Very high-resolution regional climate simulations over Scandinavia-Present climate. J. Climate, 11, 3204-3229.

Daley, R., 1991: Atmospheric Data Analysis. Cambridge University Press, 457 pp.

Davies, R., 1982: Documentation of the solar radiation parameterization in the GLAS climate model. NASA Tech. Memo. 83961, 57 pp.

Denis, B., R. Laprise, D. Caya, and J. Cote, 2002: Downscaling ability of one-way nested regional climate models. The BigBrother Experiment. Climate Dyn., 18, 627-646. 
Dirmeyer, P. A., and F. J. Zeng, 1999: An update to the distribution and treatment of vegetation and soil properties in SSiB. COLA Tech. Rep. 78, 25 pp.

Fels, S. B., and M. D. Schwarzkopf, 1975: The simplified exchange approximation. A new method for radiative transfer calculations. J. Atmos. Sci., 32, 1475-1488.

Harshvardhan, R. Davies, D. A. Randall, and T. G. Corsetti, 1987: A fast radiation parameterization for atmospheric circulation models. J. Geophys. Res., 92 (D1), 1009-1016.

Hong, S.-Y., and H.-L. Pan, 1996: Nonlocal boundary layer vertical diffusion in a medium-range forecast model. Mon. Wea. Rev., 124, 2322-2339.

Juang, H.-M., and M. Kanamitsu, 1994: The NMC nested regional spectral model. Mon. Wea. Rev., 122, 3-26.

— - S.-Y. Hong, and M. Kanamitsu, 1997: The NCEP regional spectral model: An update. Bull. Amer. Meteor. Soc., 78, 2125-2143.

Kanamaru, H., and M. Kanamitsu, 2007: Scale-selective bias correction in a downscaling of global analysis using a regional model. Mon. Wea. Rev., 135, 334-350.

Kinter, J. L., III, M. J. Fennessy, V. Krishnamurthy, and L. Marx, 2004: An evaluation of the apparent interdecadal shift in the tropical divergent circulation in the NCEP-NCAR reanalysis. J. Climate, 17, 349-361.

Leung, L. R., L. O. Mearns, F. Giorgi, and R. L. Wilby, 2003: Regional climate research. Bull. Amer. Metetor. Soc., 84, 89-95.

Liebmann, B., and C. A. Smith, 1996: Description of a complete (interpolated) outgoing longwave radiation dataset. Bull. Amer. Meteor. Soc., 77, 1275-1277.

Marbaix, P., H. Gallee, O. Brasseu, and J.-P. van Ypersele, 2003: Lateral boundary conditions in regional climate models: A detailed study of the relaxation procedure. Mon. Wea. Rev., 131, 461-479.

Mellor, G. L., and T. Yamada, 1982: Development of a turbulence closure model for geophysical fluid processes. Rev. Geophys. Space Phys., 20, 851-875.

Menendez, C. G., A. C. Saulo, and Z.-X. Li, 2001: Similation of South America wintertime climate with a nesting system. Climate Dyn., 17, 219-231.

Miguez-Macho, G., G. L. Stenchikov, and A. Robock, 2004: Spectral nudging to eliminate the effects of domain position and geometry in regional climate model simulations. J. Geophys. Res., 109, D13104, doi:10.1029/2003JD004495.

Misra, V., 2004: An evaluation of the predictability of austral summer season precipitation over South America. J. Climate, 17, 1161-1175.

_ 2005: Simulation of the intraseasonal variability of the South American Summer Monsoon. Mon. Wea. Rev., 133, 663-676.

_, and M. Kanamitsu, 2004: Anomaly nesting: A methodology to downscale seasonal climate simulations from AGCMs. $J$. Climate, 17, 3249-3262.

— , P. A. Dirmeyer, and B. P. Kirtman, 2002: A comparative study of two land surface schemes in regional climate integrations over South America. J. Geophys. Res., 107, 8080, doi:10.1029/2001JD001284.

,$- \ldots$, and -2003 : Dynamic downscaling of regional climate over South America. J. Climate, 16, 103-117.

Moorthi, S., and M. J. Suarez, 1992: Relaxed Arakawa-Schubert: A parameterization of moist convection for general circulation models. Mon. Wea. Rev., 120, 978-1002.

Nigam, S., C. Chung, and E. DeWeaver, 2000: ENSO diabatic heating in ECMWF and NCEP-NCAR reanalyses, and NCAR CCM3 simulation. J. Climate, 13, 3152-3171.

Noguer, M., R. G. Jones, and J. Murphy, 1998: Sources of systematic errors in the climatology of a nested regional climate model (RCM) over Europe. Climate Dyn., 14, 691-712.

Paegle, J. N., and K. C. Mo, 1997: Alternating wet and dry conditions over South America during summer. Mon. Wea. Rev., 125, 279-291.

Reynolds, R. W., and T. M. Smith, 1994: Improved global sea surface temperature analyses using optimum interpolation. $J$. Climate, 7, 929-948.

Risbey, J. S., and P. H. Stone, 1996: A case study of the adequacy of GCM simulations for input to regional climate change assessments. J. Climate, 9, 1441-1467.

Roads, J. O., and S.-C. Chen, 2000: Surface water and energy budgets in the NCEP regional spectral model. J. Geophys. Res., 105, 29 539-29 550.

Ropelewski, C. F., J. E. Janowiak, and M. F. Halpert, 1985: The analysis and display of real time surface climate data. Mon. Wea. Rev., 113, 1101-1107.

Staniforth, A., 1997: Regional modeling: A theoretical discussion Meteor. Atmos. Phys., 63, 15-30.

Tiedtke, M., 1984: The effect of penetrative cumulus convection on the large-scale flow in a general circulation model. Beitr. Phys. Atmos., 57, 216-239.

von Storch, H., H. Langenberg, and F. Feser, 2000: A spectral nudging technique for dynamic downscaling purposes. Mon. Wea. Rev., 128, 3664-3673.

Wang, M., J. Paegle, and S. P. DeSordi, 1999: Global variable resolution simulations of Mississippi River basin rains of summer 1993. J. Geophys. Res., 104 (D16), 19 399-19 414.

Warner, T. T., R. A. Peterson, and R. E. Treadon, 1997: A tutorial on lateral boundary conditions as a basic and potentially serious limitation to regional numerical weather prediction. Bull. Amer. Meteor. Soc., 78, 2599-2617.

Xie, P., and P. Arkin, 1996: Analysis of global monthly precipitation using guage observations, satellite estimates, and numerical model predictions. J. Climate, 9, 840-858.

Xue, Y.-K., P. J. Sellers, J. L. Kinter, and J. Shukla, 1991: A simplified biosphere model for global climate studies. J. Climate, 4, 345-364.

— F. J. Zeng, and C. A. Schlosser, 1996: SSiB and its sensitivity to soil properties. A case study using HAPEX-Mobilhy data. Global Planet. Change, 13, 183-194. 